\title{
Extracting structural information from MEH-PPV optical spectra
}

Cite as: J. Chem. Phys. 149, 044903 (2018); https://doi.org/10.1063/1.5041938

Submitted: 28 May 2018 . Accepted: 11 July 2018 . Published Online: 26 July 2018

Jonathan D. Milward, Max Marcus, Anna Köhler (D), and William Barford (D)

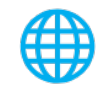

\section{ARTICLES YOU MAY BE INTERESTED IN}

Perspective: Optical spectroscopy in $\pi$-conjugated polymers and how it can be used to determine multiscale polymer structures

The Journal of Chemical Physics 146, 130902 (2017); https://doi.org/10.1063/1.4979495

Ultra-fast relaxation, decoherence, and localization of photoexcited states in $\pi$-conjugated polymers

The Journal of Chemical Physics 148, 034901 (2018); https://doi.org/10.1063/1.5009393

Theory of optical transitions in curved chromophores

The Journal of Chemical Physics 145, 12411 (2016); https://doi.org/10.1063/1.4962747

The Journal

of Chemical Physics

The Emerging Investigators Special Collection and Awards Recognizing the excellent work of early career researchers! 


\title{
Extracting structural information from MEH-PPV optical spectra
}

\author{
Jonathan D. Milward, ${ }^{1,2}$ Max Marcus, ${ }^{1,3, a), b)}$ Anna Köhler, ${ }^{4}$ and William Barford ${ }^{1,5, c)}$ \\ ${ }^{1}$ Department of Chemistry, Physical and Theoretical Chemistry Laboratory, University of Oxford, \\ Oxford OX1 3QZ, United Kingdom \\ ${ }^{2}$ University College, University of Oxford, Oxford OX1 4BH, United Kingdom \\ ${ }^{3}$ Magdalen College, University of Oxford, Oxford OX1 4AU, United Kingdom \\ ${ }^{4}$ Experimental Physics II, University of Bayreuth, 95447 Bayreuth, Germany and Bayreuth Institute \\ of Macromolecular Research (BIMF), University of Bayreuth, 95447 Bayreuth, Germany \\ ${ }^{5}$ Balliol College, University of Oxford, Oxford OX1 3BJ, United Kingdom
}

(Received 28 May 2018; accepted 11 July 2018; published online 26 July 2018)

\begin{abstract}
The Frenkel-Holstein model in the Born-Oppenheimer regime is used to interpret temperaturedependent photoluminescence spectra of solutions made with the poly(p-phenylene vinylene) derivative MEH-PPV. Using our recently developed structural optimization method and assuming only intrachain electronic coupling, we predict the structure of emissive MEH-PPV chromophores in terms of a mean torsional angle $\phi_{0}$ and its static fluctuations $\sigma_{\phi}$, assuming no cis-trans defects. This allows us to fully account for the observed changes in spectra, and the chromophore structures obtained are consistent with the known phase transition at $180 \mathrm{~K}$ between a "red" and "blue" phase. Published by AIP Publishing. https://doi.org/10.1063/1.5041938
\end{abstract}

\section{INTRODUCTION}

In previous work, solutions of the Frenkel-Holstein model under the Born-Oppenheimer approximation have provided insight into the optical spectra of conjugated polymers. ${ }^{1}$ Recently this approach was used to model the spectra of MeLPPP (poly- (2-methoxy-5-(2'-ethylhexyl)oxy 1,4phenylene vinylene) $).^{2}$ Assuming only intrachain electronic coupling and by optimizing structural parameters to obtain the best simulated vibronic peaks as compared to experiment, predictions of the changes in the polymer structure with temperature were made. In the present work, a similar procedure is performed for the poly( $p$-phenylene vinylene) derivative MEH-PPV, using solution phase photoluminescence spectra. ${ }^{3}$ This system is of particular interest because of the proposed $180 \mathrm{~K}$ phase transition between a low temperature more planarized "red" phase and a high temperature more disordered "blue" phase. This phase transition in solution is not unique to MEH-PPV, but it can be treated as a prototype system for this behavior. ${ }^{3-5}$

For the simulation of spectra, we treat individual MEHPPV polymer molecules as electronically decoupled from each other. This allows us to extract structural information from spectra with minimal computational cost. Given that aggregation may occur in the "red" phase, ${ }^{3}$ it is important to justify our neglect of interpolymer coupling.

The "red" phase spectra $(\mathrm{T} \leq 180 \mathrm{~K})$ of MEH-PPV have already been interpreted by Yamagata and co-workers. ${ }^{6}$ Assuming that polymers in the "red" phase are electronically coupled, they model the data assuming intrapolymer

\footnotetext{
a)Present address: Department of Physics, University of Warwick, Coventry CV4 7AL, United Kingdom.

b)Electronic mail: m.marcus@warwick.ac.uk

c)Electronic mail: william.barford@chem.ox.ac.uk
}

site-disorder and weak interchain coupling. This so-called H-J aggregate model has been extensively studied by Spano and co-workers. ${ }^{7,8}$ For perfectly uniform dimers at $\mathrm{T}=0 \mathrm{~K}$, the $0-0$ emission vanishes, while the $0-1$ emission is allowed via Herzberg-Teller coupling to the symmetric dimer state. As the temperature increases, the $0-0$ emission gains intensity via the thermal population of the symmetric dimer state until a crossover temperature $T_{p} \sim 2 J_{\text {inter }} / k_{B}$ is reached, ${ }^{7}$ where $J_{\text {inter }}$ is the interchain exciton transfer integral. For $T>T_{p}$, the chains are effectively electronically decoupled and $I_{00} / I_{01}$ subsequently decreases because of a decrease in intrachain exciton coherence. The predictions of the H-J aggregate model have recently been observed in two-dimensional aggregates of dye molecules. ${ }^{9}$

For disordered dimers, the $0-0$ emission does not vanish at $\mathrm{T}=0 \mathrm{~K}$ because of the breaking of symmetry. ${ }^{6}$ Nevertheless, the temperature dependence of $I_{00} / I_{01}$ is qualitatively the same as for uniform dimers: the ratio increases until $T \sim T_{p}$, whereupon it decreases (see Fig. 8 of Ref. 6).

For MEH-PPV in solution, there is no experimental evidence of a crossover in the temperature dependence of $I_{00} / I_{01}$, i.e., as illustrated in Fig. 1 (apart from fluctuations at the transition at $\sim 180 \mathrm{~K}$ ), this ratio is a monotonically decreasing function of temperature for $\mathrm{T}>100 \mathrm{~K}$, implying that $J_{\text {inter }}$ $<10 \mathrm{meV}$. (We also note that the "red" phase has the higher ratio despite being expected to have greater interpolymer coupling.) By contrast, the intrachain electronic coupling, $J_{\mathrm{intra}}$, is of the order of $2 \mathrm{eV}$ (see Table I). Since the thermal fluctuations of $J_{\text {intra }}$ themselves exceed $J_{\text {inter }}$, we believe that it is reasonable to neglect interchain electronic coupling and assume that the temperature dependence of $I_{00} / I_{01}$ arises solely from the fluctuations of the intrachain electronic coupling, noting that these may result from physical (but not electronic) interchain interactions. In other words, we consider that there can be contact interactions in which two molecules approach within the 


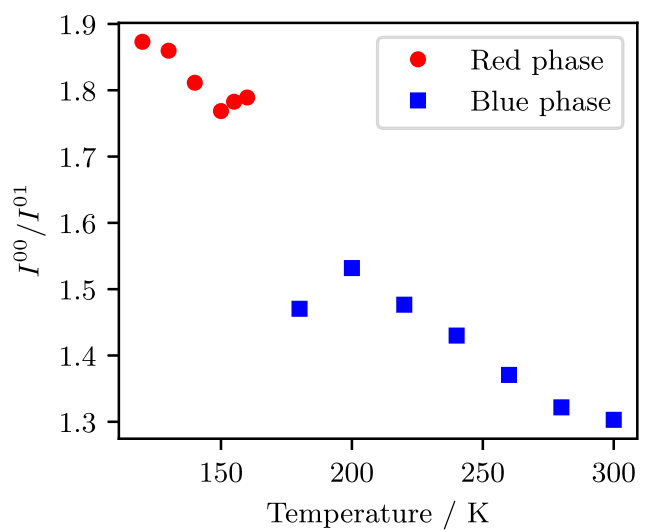

FIG. 1. Emission intensity ratio $I^{00} / I^{01}$ calculated from experimental spectra of pure "red" and pure "blue" phases of MEH-PPV (according to Ref. 3).

TABLE I. The parameters used in the Frenkel-Holstein model in the modeling of the optical spectra MEH-PPV.

\begin{tabular}{lc}
\hline \hline Parameter & Value \\
\hline Phenyl Frenkel excitation energy, $\epsilon_{p}$ & $4.5 \mathrm{eV}$ \\
Vinyl Frenkel excitation energy, $\epsilon_{v}$ & $8.59 \mathrm{eV}$ \\
Superexchange exciton transfer integral, $J_{S E}$ & $-1.68 \mathrm{eV}$ \\
Dipole-dipole exciton transfer integral, $J_{D D}$ & $-0.60 \mathrm{eV}$ \\
Phonon energy, $\hbar \omega$ & $0.19 \mathrm{eV}$ \\
Phenyl exciton-phonon coupling constant, $g_{p}$ & 3.182 \\
Vinyl exciton-phonon coupling constant, $g_{v}$ & 2.828 \\
\hline \hline
\end{tabular}

van der Waals radius, yet these do not cause electronic resonance. These observations, namely, (i) the photophysics of the "red" phase can be explained solely by intrachain electronic couplings and (ii) the transition between the "blue" phase and possibly aggregated (or more dense) "red" phase is mediated by interchain physical interactions, were also emphasised by Köhler and co-workers ${ }^{5}$ and were consistent with numerical simulations by De Leener and co-workers. ${ }^{10}$ Finally, we note that an aggregated "red" phase was observed by Peteanu and co-workers ${ }^{11,12}$ in PPV oligomers.

Section II briefly describes our methodology while Sec. III describes our modeling of the MEH-PPV spectra to obtain the structural information. We confirm a transition at $180 \mathrm{~K}$ where there is a small $\left(\sim 3^{\circ}\right)$ change in the mean torsional angle. We conclude and propose future work in Sec. IV. And the Appendix presents details of the theoretical modeling used.

\section{METHODOLOGY} limit is

The Frenkel-Holstein model in the Born-Oppenheimer

$$
\begin{aligned}
\hat{H}_{B O}= & \sum_{n=1}^{N} \epsilon_{n}|n\rangle\langle n|+\sum_{n=1}^{N-1} J(n)(|n\rangle\langle n+1|+| n+1\rangle\langle n|) \\
& -\sqrt{2} \hbar \omega \sum_{n=1}^{N} g_{n} \tilde{Q}_{n}|n\rangle\langle n|+\frac{\hbar \omega}{2} \sum_{n=1}^{N} \tilde{Q}_{n}^{2},
\end{aligned}
$$

where the index $n$ labels a moiety (i.e., a phenyl ring or vinyl dimer) and $N$ is the number of moieties in the chain. $\tilde{Q}_{n}$ is the dimensionless harmonic oscillator coordinate, defined in terms of the dimensionful coordinate, $Q_{n}$, via

$$
\tilde{Q}_{n}=\left(\frac{K}{\hbar \omega}\right)^{1 / 2} Q_{n},
$$

where $K$ is the force constant and $\omega$ is the angular frequency of the oscillation. $\epsilon_{n}$ is the Frenkel exciton excitation energy on moiety $n$.

The exciton transfer integral is

$$
J_{n}=J_{D D}+J_{S E} \cos ^{2} \phi_{n},
$$

where $J_{D D}$ and $J_{\mathrm{SE}}$ are the dipole-dipole and superexchange contributions to the transfer-integral, respectively, and $\phi_{n}$ is the torsional angle between neighboring moieties, here modeled as a Gaussian random variable with mean $\phi_{0}$ and standard deviation $\sigma_{\phi}$. This allows for a direct account of the polymer conformation on the excitonic states and associated optical properties.

The off-diagonal disorder encapsulated by $J_{n}$ causes the exciton center-of-mass wavefunction to become localized. The low energy spectrum is dominated by local exciton ground states (or LEGSs), ${ }^{13,14}$ whose spatial extent to a good approximation represents emissive chromophores. ${ }^{1}$ In our modeling, we assume that photoluminescence occurs from these LEGSs.

Invoking the Born-Oppenheimer approximation in the model (either the sufficiently small number of moieties, $N$, or $\hbar \omega \ll J$, which is the adiabatic limit; if $\hbar \omega /|J| \sim 0.1$, then $N \leq 20$ ) allows for a direct evaluation of transition intensities. Within this limit, the vibronic progression for a linear polymer is given as (ignoring energy factors) $)^{15}$

$$
\frac{A^{00}}{A^{10}}=\frac{I^{00}}{I^{10}}=\frac{\mathrm{PN}}{g^{2}}=\frac{\left(\sum_{n=1}^{N}\left|\Psi_{n}\right|^{4}\right)^{-1}}{g^{2}},
$$

where $\mathrm{PN}$ is the participation number, which is a measure of the delocalization length of the exciton and hence a direct probe of the chromophore length. $g^{2}$ is the monomeric Huang-Rhys parameter, and $\Psi_{n}$ is the wavefunction of the corresponding LEGS.

The structure of the emissive MEH-PPV chromophores is predicted using the method described in our recent paper. ${ }^{2}$ Ensembles of polymer molecules (100 molecules of 100 monomers each) were modeled with torsional angles drawn from a Gaussian distribution of mean $\phi_{0}$ and standard deviation $\sigma_{\phi}$. These parameters were optimized using the Nelder-Mead method $^{16}$ (see the Appendix) in order to minimize the difference between simulated and experimental $0-0$ and $0-1$ vibronic peaks.

The parameterization of the Frenkel-Holstein model required modification from that of PPV used previously, ${ }^{17}$ in order to give spectra consistent with experiments for any $\left(\phi_{0}\right.$, $\left.\sigma_{\phi}\right)$. This is to be expected given the differences in the chemical structure of MEH-PPV, in particular, the oxygen atoms bonded to the aromatic rings. Therefore, the phenyl excitonphonon coupling constant $g_{p}$ was raised and the phenyl exciton on-site energy $\epsilon_{p}$ was lowered, in order to produce mean torsional angles of $\sim 20^{\circ}$ at the higher temperatures, reasonably consistent with the maxima of the torsional angle distributions found in molecular dynamics simulations. ${ }^{18}$ In order to 
determine these values, gas-phase quantum chemical calculations of the first excited state were performed on MEH-PPV oligomers and the mean torsional angle and the associated force constant were found, which was used to calculate the disorder for the $80 \mathrm{~K}$ spectrum, which is the lowest temperature spectrum available. For this temperature only, these parameters were fixed and $g_{p}$ was optimized. In subsequent calculations, $g_{p}$ was assumed to be temperature-independent and held constant, while the structural parameters were optimized. Because these choices are not fully quantitative and the true torsional angle distribution is not a Gaussian as assumed, the absolute values of the structural parameters that we predict are not quantitative. The polymers were modeled with no cistrans defects, but assuming that the level of these defects is independent of temperature, this will only affect the absolute values of $\left(\phi_{0}, \sigma_{\phi}\right)$. We can, however, crucially, learn how the chromophore structure changes with temperature. The parameters of the Frenkel-Holstein model for MEH-PPV are listed in Table I.

\section{INTERPRETATION OF MEH-PPV SPECTRA}

We focus on the experimental optical spectra of MEHPPV in MTHF solution, as presented in Fig. 1 of Ref. 3. We aim to learn the extent to which the optical spectra can inform us of structural properties.

Simulated and experimental emission spectra at $160 \mathrm{~K}$ and $200 \mathrm{~K}$ are plotted in Fig. 2, as examples of the spectral fitting for the "red" and "blue" phases. The values of the structural parameters obtained from the full range of emission spectra, which according to Ref. 3 correspond to the emission from pure phases, are shown in Fig. 3. This constitutes prediction of the changing structure of emissive chromophores with temperature. There is a clear step increase in the mean torsional angle at $180 \mathrm{~K}$, supporting the notion of a phase transition. In both phases, $\phi_{0}$ decreases with decreasing temperature, showing planarization of the polymers as the temperature is lowered. ${ }^{4}$

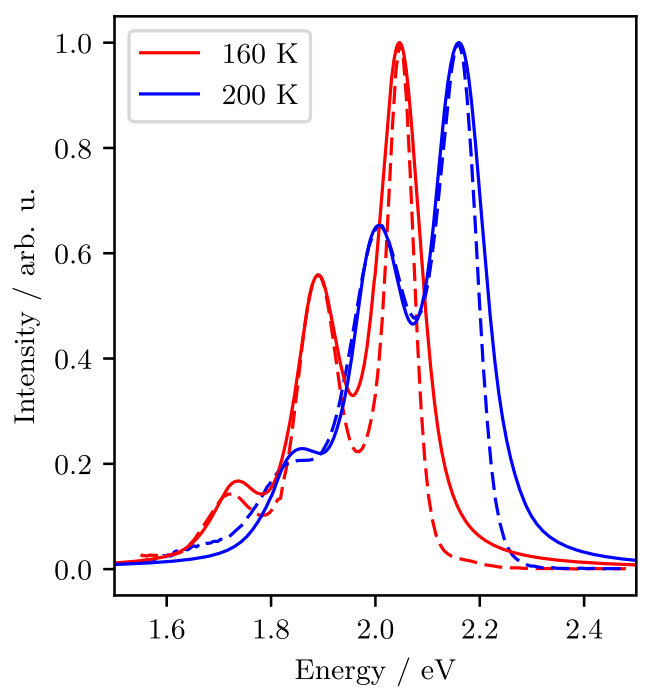

FIG. 2. Simulated (solid lines) and experimental (dashed lines) MEH-PPV emission spectra at $160 \mathrm{~K}$ and $200 \mathrm{~K}$, corresponding to pure "red" phase and pure "blue" phases, respectively. ${ }^{3}$ The spectra are normalized to the $0-0$ intensity.
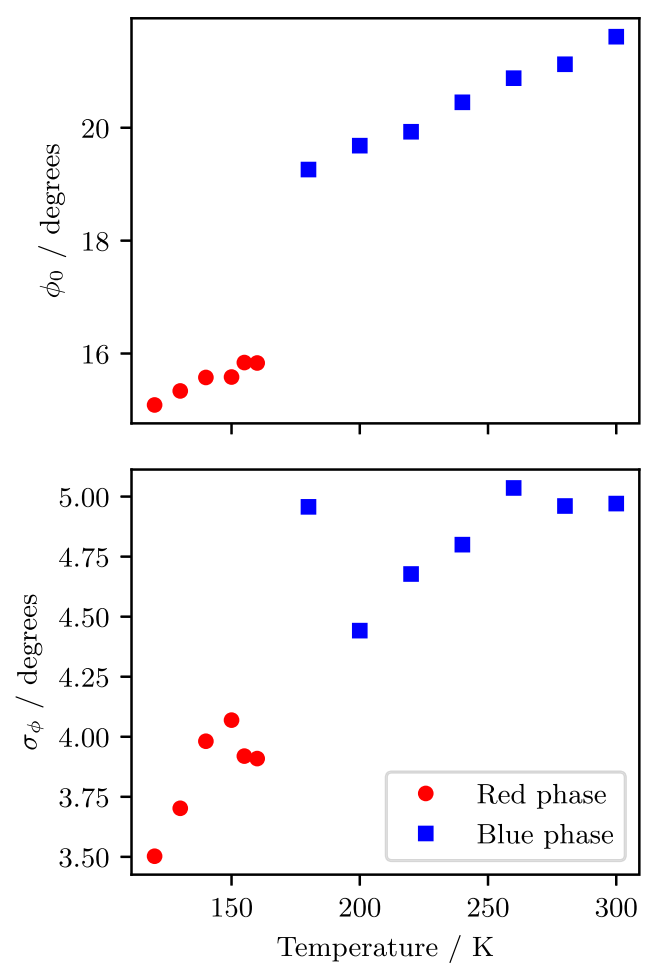

FIG. 3. Predicted mean, $\phi_{0}$, and standard deviation, $\sigma_{\phi}$, of torsional angles against temperature for the emissive chromophores of MEH-PPV, calculated from temperature-dependent photoluminescence spectra of the pure "red" and "blue" phases. ${ }^{3}$

The disorder parameter $\sigma_{\phi}$ generally increases with temperature and is greater for the "blue" phase, as expected, although the discontinuity is less clear. The changes in $\sigma_{\phi}$ depend primarily on the 0-0/0-1 ratio, explaining the similarity between the $\sigma_{\phi}$ plot and the inverse of Fig. 1. The absorption spectra were not possible to fit because of highly overlapping 0-0 and 1-0 peaks.

We can also use Eq. (4) and Fig. 1 to determine the mean size of the emitting chromophores (as defined by their PN). Using a mean value of $g^{2}=9$ implies that $\mathrm{PN} \sim 17$ at $100 \mathrm{~K}$ in the "red" phase while PN 12 at $300 \mathrm{~K}$ in the "blue" phase. These estimates are consistent with those determined in Ref. 5 via the particle-in-the-box exciton model.

\section{CONCLUSIONS AND FUTURE WORK}

In the past work, a high temperature disordered "blue" phase and a low temperature planarized "red" phase of MEHPPV have been predicted, with planarization occurring as the temperature is lowered in both phases. ${ }^{4}$ In this paper, we have explicitly interpreted the temperature dependent photoluminescence spectra using the Frenkel-Holstein model under the Born-Oppenheimer approximation. We have agreed qualitatively with the known structural changes and quantified them in terms of a mean torsional angle and its static deviations using our recently introduced structural prediction method. ${ }^{2}$ Changes in the torsional angles of uncoupled MEH-PPV molecules can fully account for the observed changes in spectra with temperature. 
It was important to justify the neglect of interpolymer coupling for MEH-PPV since the low temperature "red" phase has been described as aggregated. ${ }^{3}$ In the experimental spectra, we observe no indication that interpolymer coupling needs accounting for, in agreement with quantum chemical calculations. ${ }^{10}$ Intermolecular effects will, however, have indirect effects via the structure of polymers (particularly if aggregation occurs) and will have implications for any intermolecular diffusion.

In future work, we intend to use molecular dynamics simulations to obtain realistic MEH-PPV structures. This would allow us to understand the changes in the polymer structure in thermodynamic terms. Realistic torsional angle distributions may produce simulated spectra with line shapes that better agree with experiment, allowing more structural information to be extracted, including the frequent cases where peaks are poorly resolved. Further to this, realistic structures would facilitate simulations of exciton diffusion, the possible importance of which was highlighted in recent work. $^{2}$

\section{ACKNOWLEDGMENTS}

The authors thank Professor Heinz Bässler for fruitful discussions. J.D.M. is grateful for support from the EPSRC Centre for Doctoral training, Theory and Modelling in Chemical Science, under Grant No. EP/L015722/1, as well as University College, in the University of Oxford, through the Radcliffe Scholarship. M.M. thanks Magdalen College, in the University of Oxford, for support through a Zvi and Ofra Meitar Magdalen College Graduate Scholarship.

This paper is dedicated to the memory of its principal author, Jonathan D. Milward, who died on March 23rd, 2018.

\section{APPENDIX: OUTLINE OF THE NELDER-MEAD ALGORITHM}

The optimization algorithm used in this and previous work $^{2}$ is a simplex algorithm introduced by Nelder and Mead. ${ }^{16}$ We will give a brief outline of this algorithm.

In contrast to more conventional optimization routines, the Nelder-Mead method is gradient-free, i.e., we do not require an approximation of the gradient at a given point to find the direction in which to take the next step. This significantly reduces the computational cost, and most steps will only require the evaluation of the cost function at two distinct points.

In this work, we have chosen the parameters $\phi_{0}$ and $\sigma_{\phi}$ as the variables and have defined a cost function, $F$, as the (dimensionless) square of the distance of the simulated 0-0 and 0-1 vibronic peaks from their experimental counterparts as

$$
F\left(\phi_{0}, \sigma_{\phi}\right)=\left(1-\frac{E_{\text {calc }}^{00}}{E_{\text {exp }}^{00}}\right)^{2}+\left(1-\frac{E_{\text {calc }}^{01}}{E_{\text {calc }}^{01}}\right)^{2}+\left(R_{\text {exp }}-R_{\text {calc }}\right)^{2},
$$

where $R$ is the $0-0 / 0-1$ intensity ratio. Other cost functions can be chosen, but the results we obtained show that this choice is sufficient for the systems we have considered.
The algorithm then iterates in the following way until termination:

1. Initialization: In an $n$-dimensional optimization space (where $n$ is the number of parameters in the cost function), find $n+1$ vertices, $\left\{\mathbf{x}_{i}\right\}$, and evaluate the cost function, $\left\{F_{i}\right\}$. If one vertex is given as input, call it $\mathbf{x}_{0}$ (as is usually the case- the initial guess), then the remaining vertices are often chosen such that the initial simplex is either right-angled in $\mathbf{x}_{0}$ along the coordinate axes or such that the simplex is equilateral.

2. Ordering: Out of the cost function values generated, determine the best, $F_{a}$, worst, $F_{z}$, and second-worst, $F_{y}$, functional value, i.e.,

$$
F_{a}=\min _{i} F_{i}, \quad F_{z}=\max _{i} F_{i}, \quad F_{y}=\max _{i \neq z} F_{i},
$$

in the case of minimization.

3. Centroid: Calculate the center-of-mass of the best side within the simplex, i.e., the side opposite the worst vertex,

$$
\mathbf{c}=\frac{1}{n} \sum_{i \neq z} \mathbf{x}_{i}
$$

4. Transformation: Create a new simplex by replacing the first vertex with a new one. This is done either by reflection, expansion, or contraction. If neither provides a better simplex, shrink the current simplex toward the best vertex.

5. Termination: Terminate the procedure (steps 2-4) if some criterion is reached. Either the difference between $F_{a}$ and $F_{z}$ is below a certain threshold or the change in the simplex is below a certain threshold. Other termination criteria can be introduced if necessary.

The transformation step is the most crucial as this is the step in which new vertices are introduced. If we consider an optimization in two parameters (as is the case in this work), then the simplex will be a general triangle with vertices $\mathbf{x}_{a}$, $\mathbf{x}_{y}, \mathbf{x}_{z}$. The centroid will be half-way between $\mathbf{x}_{a}$ and $\mathbf{x}_{y}$ on the side opposite $\mathbf{x}_{z}$ (see Fig. 4). Reflection will generate a new vertex, $\mathbf{x}_{r}$ at $\mathbf{x}_{r}=\mathbf{c}+\alpha\left(\mathbf{c}-\mathbf{x}_{z}\right)$. If the new vertex has a value such that $F_{a} \leq F_{r}<F_{y}$, then this is accepted and the new simplex is found. If this is not the case, expansion or contraction is performed, depending on the relation of the cost function values. If the new functional value is less than the previously best value, i.e., $F_{r}<F_{a}$, then a new vertex is calculated expanding the simplex in that direction (as it appears to be a good search direction). Calculate $\mathbf{x}_{e}=\mathbf{c}+\gamma\left(\mathbf{x}_{r}-\mathbf{c}\right)$ and the associated cost function value. If $F_{e}<F_{r}$, then accept the new vertex, and if $F_{r} \leq F_{e}$, then keep the reflected vertex. The choice of $<$ and $\leq$ here can be crucial. The way it is given here, the simplex will be kept small by choosing the smaller simplex for equal cost function values, while it can be advantageous to keep the simplex large, in which case the relations have to be swapped. If, after reflection, it is found that $F_{r} \geq F_{y}$, then contraction is performed. A new vertex is found as that will make the simplex smaller. For this, two choices can be made: either the new simplex lies within the old simplex, or not. If $F_{z}>F_{r} \geq F_{y}$, then the search direction is a good one and the new simplex will be located outside the old one. In this case, calculate $\mathbf{x}_{c}=\mathbf{c}+\beta\left(\mathbf{x}_{r}-\mathbf{c}\right)$. If $F_{c}<F_{r}$, then accept the new 


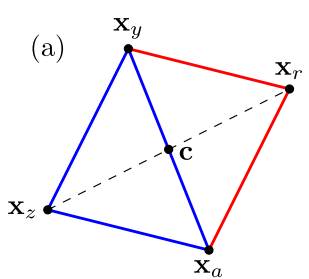

reflection

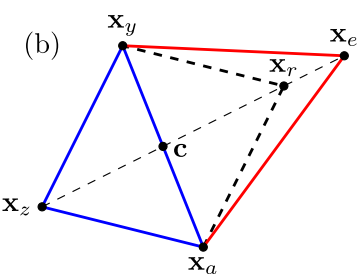

expansion

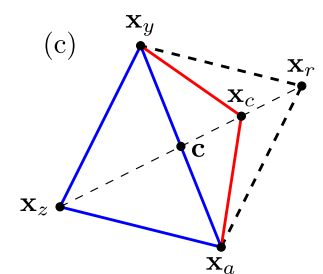

outside-

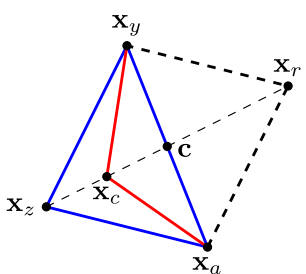

inside-

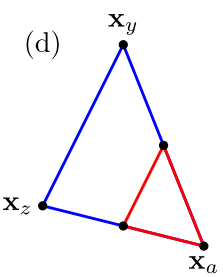

shrinking

FIG. 4. Schematic depiction of procedures for generating a new simplex in the Nelder-Mead method in a two-dimensional parameter space. Reflection (a) is performed first (and always) to generate a new simplex (red) from an old one (blue) and then, depending on the cost function value at $\mathbf{x}_{r}$, either expansion (b) or contraction (c) (inside or outside) is performed. If neither leads to a new and better simplex, the original simplex is shrunk (d).

vertex and continue. If, however, after reflection it is found that $F_{r} \geq F_{z}$, then a better point is within the simplex. In this case, calculate the contraction point as $\mathbf{x}_{c}=\mathbf{c}+\beta\left(\mathbf{x}_{z}-\mathbf{c}\right)$. If the new cost function value is better than $F_{z}$, accept the new vertex and continue. If neither of these steps leads to an improved vertex, the simplex is contracted toward the best vertex as $\mathbf{x}_{i}=\mathbf{x}_{a}+\delta\left(\mathbf{x}_{i}-\mathbf{x}_{a}\right): \quad i \neq a$. The parameters $\alpha, \beta$, $\gamma, \delta$ are similar to the step length in gradient methods and can be chosen before starting the algorithm.

In practice, the shrink operation rarely to never occurs, and as a result for every step at most, two new vertices have to be computed, significantly reducing the computational cost compared to gradient methods.

${ }^{1}$ W. Barford and M. Marcus, J. Chem. Phys. 146, 130902 (2017).

${ }^{2}$ M. Marcus, J. D. Milward, A. Köhler, and W. Barford, J. Phys. Chem. A 122, 3621 (2018).

${ }^{3}$ T. Unger, F. Panzer, C. Consani, F. Koch, T. Brixner, H. Bässler, and A. Köhler, ACS Macro Lett. 4, 412 (2015).

${ }^{4}$ F. Panzer, H. Bässler, and A. Köhler, J. Phys. Chem. Lett. 8, 114 (2017).

${ }^{5}$ A. Köhler, S. T. Hoffmann, and H. Bässler, J. Am. Chem. Soc. 134, 11594 (2012).
${ }^{6}$ H. Yamagata, N. J. Hestand, F. C. Spano, A. Köhler, C. Scharsich, S. Hoffmann, and H. Bässler, J. Chem. Phys. 139, 114903 (2013).

${ }^{7}$ H. Yamagata and F. C. Spano, J. Chem. Phys. 136, 184901 (2012).

${ }^{8}$ N. J. Hestand and F. C. Spano, "Expanded Theory of H- and J-Molecular Aggregates: The Effects of Vibronic Coupling and Intermolecular Charge Transfer," Chem. Rev. (published online).

${ }^{9}$ Al. Eisfeld, C. Marquardt, A. Paulheim, and M. Sokolowski, Phys. Rev. Lett. 119, 097402 (2017).

${ }^{10}$ C. De Leener, E. Hennebicq, J. C. Sancho-Garcia, and D. J. Beljonne, Phys. Chem. B 113, 1311 (2009).

${ }^{11}$ L. A. Peteanu, G. A. Sherwood, J. H. Werner, A. P. Shreve, and T. M. J. Smith, Phys. Chem. C 115, 15607 (2011).

${ }^{12}$ G. A. Sherwood, R. Cheng, R. M. Smith, J. H. Werner, A. P. Shreve, L. A. Peteanu, and J. Wildeman, J. Phys. Chem. C 113, 18851 (2009).

${ }^{13}$ A. V. Malyshev and V. A. Malyshev, "Statistics of low energy levels of a one-dimensional weakly localized Frenkel exciton: A numerical study," Phys. Rev. B: Condens. Matter Mater. Phys. 63, 195111 (2001).

${ }^{14}$ D. V. Makhov and W. Barford, "Local exciton ground states in disordered polymers," Phys. Rev. B: Condens. Matter Mater. Phys. 81, 165201 (2010).

${ }^{15}$ W. Barford and M. Marcus, J. Chem. Phys. 141, 164101 (2014).

${ }^{16}$ J. A. Nelder and R. Mead, Comput. J. 7, 308 (1965).

${ }^{17}$ M. Marcus, O. R. Tozer, and W. Barford, J. Chem. Phys. 141, 164102 (2014).

${ }^{18}$ K. H. Dubay, M. L. Hall, T. F. Hughes, C. Wu, D. R. Reichman, and R. A. Friesner, J. Chem. Theory Comput. 8, 4556 (2012). 